Rabaska

Revue d'ethnologie de l'Amérique française

\title{
Le Patrimoine culturel de l'Université de Poitiers, sa mise à disposition et sa valorisation : le fonds Valière
}

\section{Catherine Robert}

Volume 9, 2011

URI : https://id.erudit.org/iderudit/1005909ar

DOI : https://doi.org/10.7202/1005909ar

Aller au sommaire du numéro

Éditeur(s)

Société québécoise d'ethnologie

ISSN

1703-7433 (imprimé)

1916-7350 (numérique)

Découvrir la revue

Citer ce compte rendu

Robert, C. (2011). Compte rendu de [Le Patrimoine culturel de l'Université de Poitiers, sa mise à disposition et sa valorisation : le fonds Valière]. Rabaska, 9 , 236-240. https://doi.org/10.7202/1005909ar d'utilisation que vous pouvez consulter en ligne. 


\section{Colloques et rassemblements}

\section{Le Patrimoine culturel de l'Université de Poitiers, sa mise à disposition et sa valorisation : le fonds Valière}

CATHERINe RoberT ${ }^{1}$

À l'occasion du transfert du fonds Valière, à partir du 19 novembre 2010, au Centre de documentation de la Maison des sciences de l'Homme et de la société, une journée d'études a été organisée à Poitiers le 24 novembre 2010 pour rendre hommage au travail de collecte orale de Michèle Gardré-Valière et de son époux Michel Valière, dans la perspective d'une coopération de recherche entre des universités nord-américaines et le laboratoire Мiммос (Mémoire, identités, marginalités dans le monde occidental contemporain) de l'Université de Poitiers, dirigé par madame Susan Finding, dans le dessein d'une valorisation scientifique de cet important fonds d'archives sonores de près de quarante années.

Rappelant la disponibilité d'esprit et les qualités humaines de ces deux chercheurs (unis à la ville comme dans leurs travaux de terrain), Marlène Belly, ethnomusicologue, enseignante à l'Université de Poitiers et membre du Mimmoc, a accueilli un public attentif, très sensibilisé à la collecte ethnographique et particulièrement intéressé par la valorisation des documents sonores inédits, venu de divers horizons tant géographiques (Bretagne, Pays de Loire, Poitou-Charentes) que disciplinaires (ethnologues, historiens, linguistes, sociologues...). Ainsi, au fil de la journée, des intervenants ont présenté successivement leurs propres actions et expériences dans le domaine de l'oral afin de délimiter le champ de réflexion autour de la question centrale, celle de la mise à la disposition des publics et notamment des chercheurs des documents oraux et audiovisuels inédits.

En premier lieu, Charles Quimbert, directeur de l'association bretonne Dastum, créée en 1972, a rappelé le souci de rassembler pour le transmettre

1. Diplômée en ethnologie de l'École des hautes études en sciences sociales, Paris, 1985 ; directrice de la Médiathèque municipale de Saint-Junien (Haute-Vienne) ; présidente de l'Association régionale pour la promotion de l'ethnologie en Poitou-Charentes (ARPE). 
le patrimoine culturel de Bretagne et surtout le patrimoine immatériel et s'interroge sur la place accordée au passé et à la mémoire dans la construction de l'enfant. En outre cette culture doit être connue et développée au titre même du maintien de la diversité. Il s'agit donc, dans une perspective de sauvegarde de collecter et d'inventorier des répertoires, de se rapprocher des anciens collecteurs, revalorisant ainsi les relations humaines entre générations. Du point de vue technique et s'agissant de conservation, la numérisation s'impose pour la communication et la diffusion en réseau, en veillant à la fois à la compatibilité des appareils entre eux et, du point de vue déontologique, au respect des droits moraux de chacun (témoins et informateurs, collecteurs et institutions).

Puis, Arnaud Caillé, directeur de 1'UPCP ${ }^{2}$-Métive, à Parthenay (DeuxSèvres), exposa un bref historique de cette union régionale d'associations, née en 1968, à l'initiative d'André Pacher et de Michel Valière, qui a conduit un travail de valorisation des cultures et savoirs populaires. Les séries de collectes, individuelles ou associatives ayant trait à la musique, au chant, aux danses, aux contes du Poitou-Charentes et de la Vendée constituent une importante documentation, fonds géré par le CERDo (Centre d'études, de recherche et de documentation sur l'oralité), en cours de numérisation dans le dessein d'une réappropriation par la population contemporaine. Cette structure organise à cette fin l'accès aux collectes par le biais d'Internet. Ajoutons, et ce n'est pas son moindre intérêt, que ce même fonds constitue un socle de connaissances qui nourrit la création artistique et culturelle pour le spectacle vivant. Arnaud Caillé souligne également la complémentarité des différentes collectes réalisées dans le Centre-Ouest de la France.

Justement, à ce propos, Jean-Pierre Bertrand, président d'Ethnodoc, évoque les débuts d'une action associative en terre vendéenne à partir de 1970 qui donna lieu, à partir de 1973, à de nombreuses collectes d'images, de sons, d'objets, aboutissant à la création d'un "musée paysan » sur une thématique inspirée par les travaux de Van Gennep, « Du berceau à la tombe ». Ces initiatives ont permis la mise en place à partir de 1989 de classespatrimoine. De 2000 à 2004, a été organisée avec le concours des pouvoirs publics et le soutien de l'Europe (Programme Leader 2) une collecte sur l'ensemble des communes du département de Vendée, ce qui permit en outre de développer un partenariat avec l'Université de Nantes, mais aussi avec le Musée de l'Homme. Toutes les collectes engrangées sont organisées dans un catalogue en ligne dont les notices sont consultables sur un réseau de vingtquatre sites.

Toujours dans ce même esprit de mise en commun et de communication au plus grand nombre de la documentation sonore et audiovisuelle, Pierre-

2. UPCP : Union pour la culture populaire en Poitou-Charentes et Vendée. 
Olivier Laulanné, directeur de la FAMDT (Fédération des associations de musiques et danses traditionnelles), a présenté le portail collectif du patrimoine oral réalisé avec le soutien de la BNF (Bibliothèque nationale de France). En effet, depuis 1999, la FAMDT a constitué un réseau pour sauvegarder, documenter, valoriser les documents collectés dans les régions. Celui-ci compte actuellement cinq centres de documentation, et il est reconnu comme un «pôle associé » de la BNF. Pour une meilleure consultation, les bases de données ont été mises au même format à l'aide du protocole $\mathrm{OAI}_{\mathrm{AMH}}{ }^{3}$. Précisons que Dastum est membre de ce pôle associé avec quatre autres centres de documentation : le CERDO (UPCP-Métive), le Conservatoire occitan à Toulouse, la Maison du patrimoine oral de Bourgogne, la Phonothèque de la Maison des sciences de l'homme à Aix-en-Provence. Ce pôle associé est à l'initiative du Portail du patrimoine oral (PPO), premier portail consacré au patrimoine oral de France ${ }^{4}$.

Michel Valière initialement pressenti pour une légitime intervention, présent mais visiblement trop ému par cette journée très ciblée, fut remplacé par son épouse, Michèle Gardré-Valière qui évoqua les prémices de leur action qu'elle situa vers 1963-1964, alors qu'ils étaient jeunes enseignants au Lycée André-Theuriet à Civray (Vienne), leurs premières collectes sonores n'ayant été réalisées qu'à partir de 1965, à la suite de l'acquisition d'un premier magnétophone à rubans (quatre pistes) et de la rencontre avec l'Association française des chasseurs de sons (AfCs), puis de la Société d'études ethnologiques et folkloriques du Centre-Ouest (SÉFCO). Elles se sont poursuivies jusqu'au début du XxI ${ }^{\mathrm{e}}$ siècle. Il s'agit donc aujourd'hui de mieux organiser, en particulier, l'accès du public des chercheurs et donc de rendre perceptible et disponible cet ensemble d' ' archives sonores 》 à caractère ethnographique, mais aussi linguistique qui ont été constituées avec des partenariats divers dont la Phonothèque nationale à Paris, l'Office audiovisuel et la Bibliothèque universitaire (aujourd'hui Service commun de documentation) de l'Université de Poitiers, et désormais en partenariat avec la Maison des sciences de l'Homme et de la société. Or, la généralisation, le développement de nouvelles technologies de communication offrent des voies hier inconnues, voire impensables, à la fois pour la conservation des archives orales et pour leur exploitation par les publics les plus variés. Cependant, elles exigent, pour ce faire, une excellente connaissance des sources qui les constituent, de leur statut juridique comme des usages et des coûts engendrés par leur gestion et leur utilisation.

\footnotetext{
3. Open Archives Initiative-Protocol for Metadata harvesting.

4. http://www.portaildupatrimoineoral.org.
} 
Selon un rapport d'Aurélie Melin ${ }^{5}$, collectrice de mémoire (c'est actuellement un nouveau métier !), réalisé à la demande du laboratoire GerHICO de l'Université de Poitiers, le fonds rassemble environ mille quatre cents rubans magnétiques dont seulement quelques-uns ne sont pas datés et ne peuvent donc être situés chronologiquement avec certitude. Ils ont été enregistrés au cours d'enquêtes, à partir d'entretiens directifs ou semi-directifs, selon la méthode de recueil des récits de vie, où s'inscrivent des répertoires de contes, de chansons, de danses assez généralement remis en contexte par les informateurs - ceux-ci, le plus souvent « âgés »-dans la mesure où l'entretien permet de laisser glisser la parole et de réveiller la mémoire familiale. Les différentes « aires d'enquête » concernent plusieurs territoires de l'Hexagone, et en particulier Poitou-Charentes ainsi que plusieurs territoires de langue d'oc (Agenais, Languedoc, Limousin), de Bretagne, de Normandie... Hors frontières, quelques incursions au Tras Os Montes au Portugal (1970), en Wallonie (1972) et jusqu'en Hongrie (1975) dans le cadre de missions spécifiques ont permis de mettre en perspective les différentes cultures.

Michèle Gardré-Valière, partageant son émotion avec son époux, raconte le plaisir qu'elle éprouvait à écouter et réentendre les interprétations de ce que les informatrices dénommaient alors « complaintes » (complaintes amoureuses au sens du XVI ${ }^{e}$ siècle). La première qui les ait touchés fut celle d'une vieille dame: «Dedans Paris 1'y a une gentille brunette... » Ainsi commença alors pour Michel une longue immersion d'ethnologie participante dans le Pays des Brandes. Il revenait de ses visites avec un enthousiasme communicatif, devenu légendaire. Aujourd'hui, on le désignerait comme un geek [maniaque] du magnétophone, de l'enregistrement sonore et surtout de l'interaction avec les milieux qu'il fréquentait : paysans, salariés agricoles, lingères, artisans ruraux, étalonniers, vétérinaires... Cette passion en fit un enquêteur insatiable, exceptionnel selon la linguiste Henriette Walter, et de ses enquêtes, "des modèles du genre ${ }^{6}$ ». Ethnolinguiste, il invitait ses interlocuteurs à reconnaître dans ce qu'ils nommaient «patois » ou même « français écorché », un véritable parler d'oïl.

D'un autre point de vue, Jean-Christophe Valière, acousticien, professeur des universités (Institut P') à Poitiers, a rappelé ses propres travaux de thèse sur la restauration des enregistrements sonores, où il a cherché comment évaluer les besoins de restauration, mettant ainsi l'acoustique au service du patrimoine. Il a souligné, pour avoir vécu sa constitution, la cohérence

5. Aurélie Melin, Histoire de l'agriculture du département de la Vienne (86) : l'examen du fonds Michel Valière, Bibliothèque universitaire de Poitiers, Poitiers, Gerhico, Université de Poitiers, 2000 (non paginé, multicopié).

6. Henriette Walter, Enquête phonologique et variétés régionales du français, Paris, Presses universitaires de France, 1982, p. 72. 
géographique, temporelle et technique du fonds Valière et s'interroge sur la manière de conserver d'autant, qu'on le veuille ou non, que tout support matériel est d'avance condamné. Aussi la numérisation s'impose-t-elle, mais celle-ci n'est absolument pas suffisante. En revanche, par des moyens de sauvegarde automatique, avec redondance par des mises en réseau, par exemple, il est permis d'espérer une pérennisation des fonds sonores.

Rodolphe Defiolle a présenté le Service de documentation de la Mshs de Poitiers dont la phonothèque de recherche, créée à son initiative. Le fonds Valière en constitue le premier dépôt d'archives sonores inédites qui prend place au côté de sept autres fonds hébergés, répartis sur plus de 500 mètres carrés. Cette phonothèque devrait établir des liens constructifs avec d'autres centres de ressources sonores dans une dynamique d'accroissement des fonds. Elle entend partager en réseau ses outils de travail et établir les partenariats nécessaires.

Le directeur de l'Institut franco-canadien, André Magord, a rappelé que plusieurs universités nord-américaines connaissent déjà l'existence du fonds Valière et il tient à encourager sa valorisation par la numérisation en particulier, étape nécessaire pour sa connaissance et son exploitation en s'appuyant sur une analyse pluriculturelle dans le cadre d'une société postmoderne. Une prise en charge professionnelle de ce fonds (traitement documentaire, conservation, autorisations de consultation...) en faciliterait l'usage universitaire.

Enfin, Marlène Belly, en sa qualité d'ethnomusicologue, initiatrice de cette journée d'études dans la perspective de valorisation du fonds Valière, a rappelé l'intérêt des collectes orales pour la connaissance des répertoires de chansons. Elle s'inscrit dans une approche comparative autour de l'axe France/ Canada. L'étude du fonds, dans ses constantes mais aussi dans ses spécificités spatiales et temporelles, permettrait de préciser les contours d'une mémoire collective et identitaire, l'oralité étant, dans le domaine de la chanson, une des clefs essentielles de la transmission.

Cette journée très studieuse n'est toutefois qu'un point de départ pour la connaissance d'un fonds que je sais particulièrement riche et original pour y avoir été associée dans le cadre de travaux de recherche et d'édition. 